which bloody or water-clear fluid was contained. Membrane of dural side was thinner than that of bone side. Concerning the microscopic appearance of those membranes, there was no difference from that of subdural hematoma.

Accordingly, it was summarized that enclosing neomembrane was a product of the proliferative reaction between the dura mater and epidural or subdural blood clot.

\title{
33. Neurological Consideration on the Chronic Subdural Hematoma
}

\author{
Teruyoshi Hashiba and Ikuro TAKADA \\ Dept. of Neurological Surgery, Sapporo Medical College and Hospital
}

\begin{abstract}
Many researches has been doing on the chronic subdural hematoma by neurosurgeon and neuropathologist in past 4 centuries but the majority of the studies had done on the pathological etiology, diagnostic procedure and operative treatment and the detail studies on their neurological symptoms and signs to establish the clinical diagnosis are very few.

The authors had reported the 7 groups of neurological symptoms showed in admitted time from neurological analysis of 51 patients who had treated in authors clinic and correlation between these groups and angiographic and operative findings.

The 7 groups are as follow:

Group 1 who has mental and character changes, and personality disorders (15 cases).

Group 2 who has symptom of increased intracranial pressure (14 cases).

Group 3 who has cortical motor disorders.

Group 4 which is combined case of group 1 and group 2.

Group 5 which is combined case of group 1 and group 3 .

Group 6 which is combined case of group 2 and group 3 .

Group 7 which is combined case of group 1, group 2 and group 3.

The cases who have mental and personality change alone or combined with other symptom are 30 cases, $58.8 \%$ of all cases and this symptom is very valuable in clinical diagnosis of chronic subdural hematoma. On the other hand, high aged patients, the case who has localized hematoma in the frontal area and the case who showed low CSF pressure are observed in patients of group 1 .
\end{abstract}

